\title{
Laser Assisted Smile Designing
}

\author{
${ }^{1}$ Monica Gupta, ${ }^{2}$ Suchetan Pradhan \\ ${ }^{1}$ Consultant, Department of Prosthodontics and Laser Dentistry, Pradhan Dental Center, Mumbai, Maharashtra, India \\ ${ }^{2}$ Co-worker, Aachen University, Germany, Founder President, Indian Academy of Laser Dentistry, Director, Department of Laser Dentistry \\ Manipal, Professor, Department of Implantology, SS Dental College, Hyderabad, Andhra Pradesh, India
}

Correspondence: Monica Gupta, Consultant, Department of Prosthodontics and Laser Dentistry, Pradhan Dental Center, Mumbai Maharashtra, India, e-mail: drmonicagupta@hotmail.com

\section{ABSTRACT}

Advances in dental ceramics and advent of laser dentistry has simplified esthetic rehabilitation with predictable results. Relatively painless procedures and rapid healing as compared to conventional techniques make it a more patient friendly approach.

Keywords: Dental lasers, Smile designing.

\section{INTRODUCTION}

Advances in all ceramic materials allow practitioners to restore function and esthetics using conservative and biologically sound methods as well as promoting long-term oral health.

To design the optimal outcome for a patient during esthetic enhancement, the restorative dentist must seek to create a symmetrical and harmonious relationship between the lips, gingival architecture and the positions of the natural dentate forms. ${ }^{1,2}$

Dental lasers enable painless, minimally invasive, more precise and significantly more efficient completion of procedures associated with esthetic and restorative dentistry. ${ }^{3,4}$ Gingival and/or osseous recontouring can be easily done to establish a harmonious and esthetic soft tissue profile. ${ }^{5}$ Patients typically experience little postoperative discomfort when a laser is used with faster healing resulting in greater patient acceptance.

\section{CASE REPORT}

A 20-year-old female patient came to our practice with a chief complaint of malaligned teeth and desired a more esthetic smile. She also desired results in a short period of time.

\section{Examination}

Clinical examination showed malposed anterior teeth with crowding, caries and staining. Right canine was labially placed, and left lateral incisor was tipped labially. The lower anteriors exhibited mild crowding and both right and left canines were labially placed. Overall gingival asymmetry was observed (Fig. 3). Orthodontics was advised but due to lack of time, the patient was not willing for the same and opted for the quicker technique of smile designing.
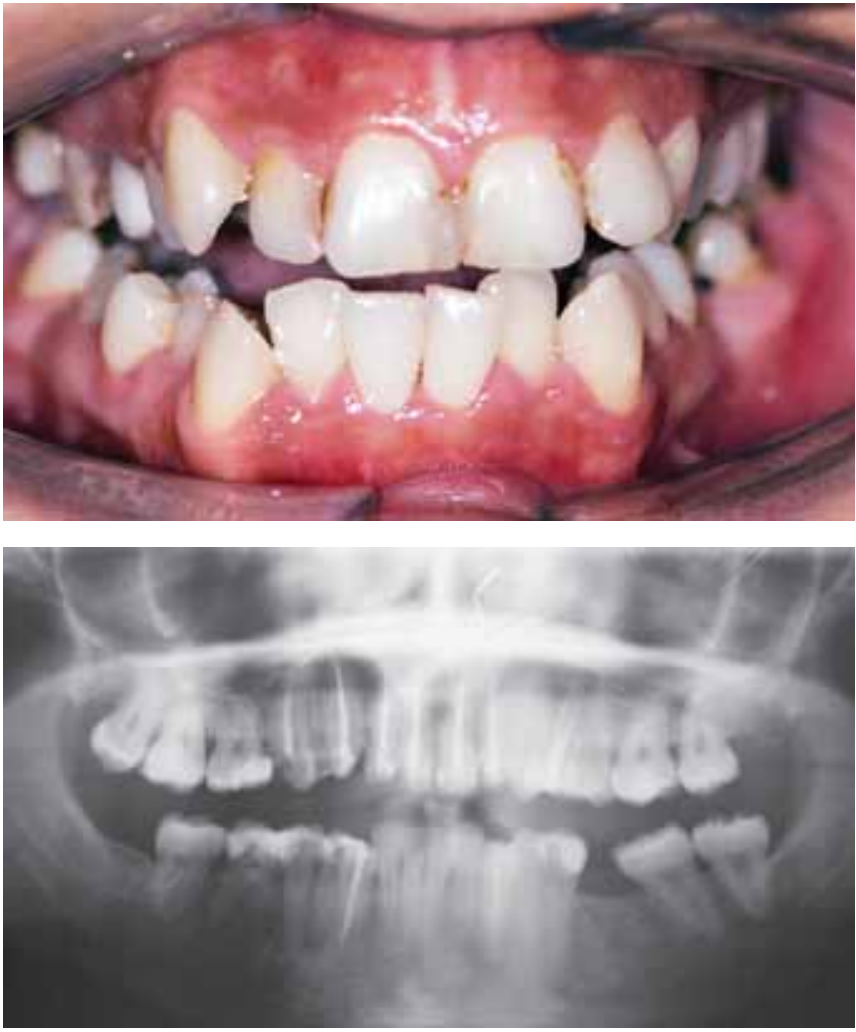

Fig. 1: Preoperative intraoral view and orthopantomogram

\section{Treatment Plan}

Impressions for study models and preoperative photographs were taken. After careful analysis of the study models, the treatment was planned.

The treatment plan included all ceramic veneers and crowns with gingival recontouring. Endodontic treatment was also required. 
The treatment plan was divided into two phases in which the maxillary arch would be restored first followed by the mandibular arch.

In the first phase, endodontic treatment was carried out in teeth nos.11-14, 21, 22, 24, and 33-44. Veneer preparation was carried out on 11, 12, 14, 21, 23 and 24 (pressed ceramic, IPS Empress). Crown preparation was done on 13 and 22 to receive all ceramic crowns (Zirconia Crowns, LAVA Zirconia, 3M). For ease of adjusting occlusion, the cusp tips of right and left mandibular canines were recontoured (Fig. 2). Impressions were made in addition silicone (Aquasil Putty and Light Body, Dentsply), bite registration records and shade were sent to the laboratory. Temporization (Charisma A1, Heraeus Kulzer) was done and patient was recalled after 2 days for try in and cementation.

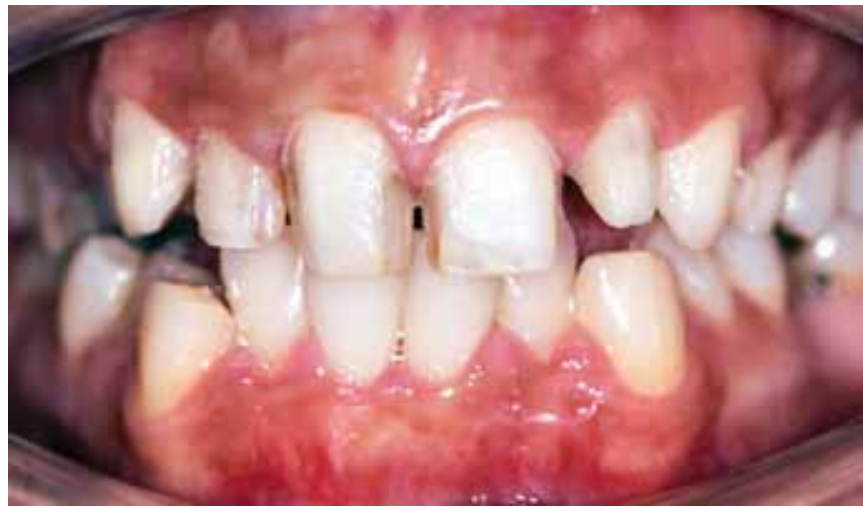

Fig. 2: Tooth preparation for all ceramic crowns and veneers. Note that the modified cusp tips of both mandibular canines

After 2 days, the temporary restorations were removed, teeth cleaned and restorations were tried in to check for fit, shape, color and contour. Veneers were cemented with light cure resin cement (Variolink, Ivoclar Vivadent) and crowns were cemented with resin modified glass ionomer cement (RelyX Luting 2, 3M ESPE) (Fig. 3). Excess was removed, contacts were checked and occlusion was adjusted.

In the second phase of treatment, the mandibular teeth were prepared for crowns to correct the alignment of the teeth. As was discussed earlier, both mandibular canines were prominently placed labially, incisors exhibited mild crowding,

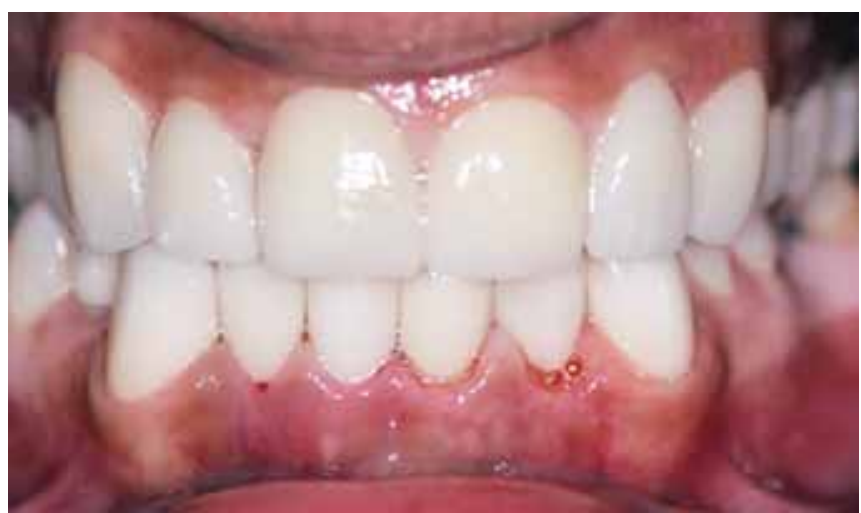

Fig. 3: Postoperative healing following closed flap osseous recontouring in lower anterior region right first premolar was placed lingually giving the appearance of an edentulous area and gingival profile was irregular.

On careful examination of the biological width, gingival and osseous recontouring was carried out in the region of the incisors using $2780 \mathrm{~nm}$, Er,Cr:YSGG laser (Waterlase MD, Biolase) (Fig. 4). Bone tapping was carried out during the osseous recontouring procedure to ensure that ledges were not created.

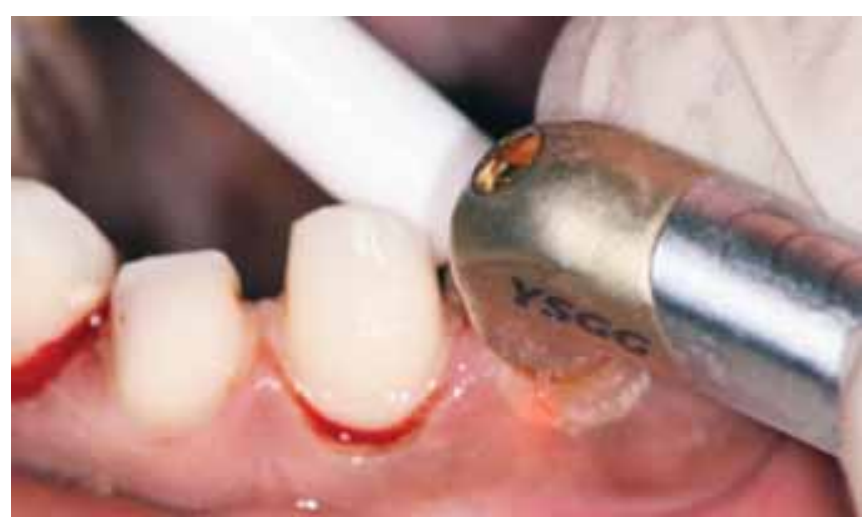

Fig. 4: Gingival and osseous recontouring with the erbium laser

The laser parameters were as follows:

- For soft tissue recontouring-1 W, $20 \mathrm{~Hz}, 8 \%$ water, 11\% air

- For hard tissue recontouring-2.5 W, 25 Hz, 30\% water, $30 \%$ air.

Crown preparations for all ceramic zirconia crowns (Zirconia Crowns, LAVA Zirconia, 3M) were carried out from right first premolar to left canine. Impressions were made in addition silicone impression material (Aquasil Putty and Light Body, Dentsply), bite recorded and shade was sent to the laboratory for fabrication. Temporization was done (Charisma A1, Heraeus Kulzer) and patient was recalled after 2 days for try in procedure and cementation.

After 2 days, healing of the tissues was excellent, temporaries were removed, teeth cleaned and restorations were checked for fit, shape, color and contour. Crowns were cemented with resin modified glass ionomer luting cement (RelyX Luting 2, 3M ESPE). Excess was removed, contacts were checked and occlusion was adjusted (Fig. 5) in centric and eccentric contacts (Fig. 6).

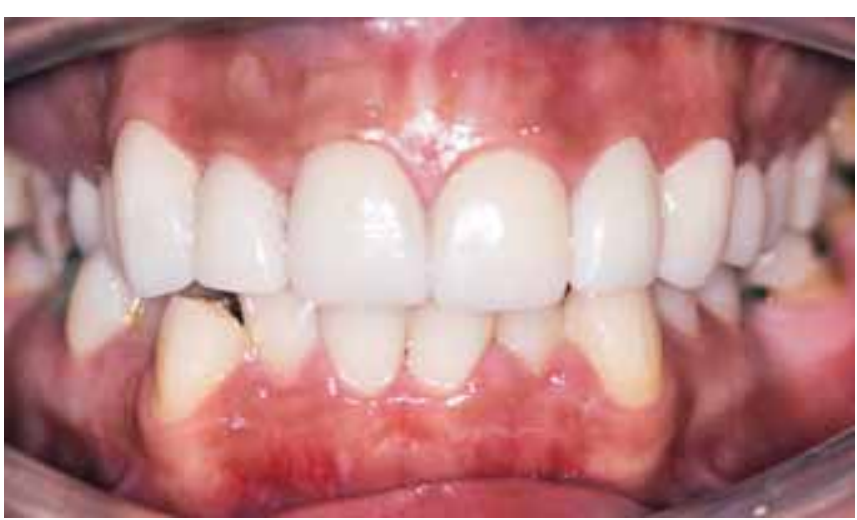

Fig. 5: Maxillary restorations cemented 


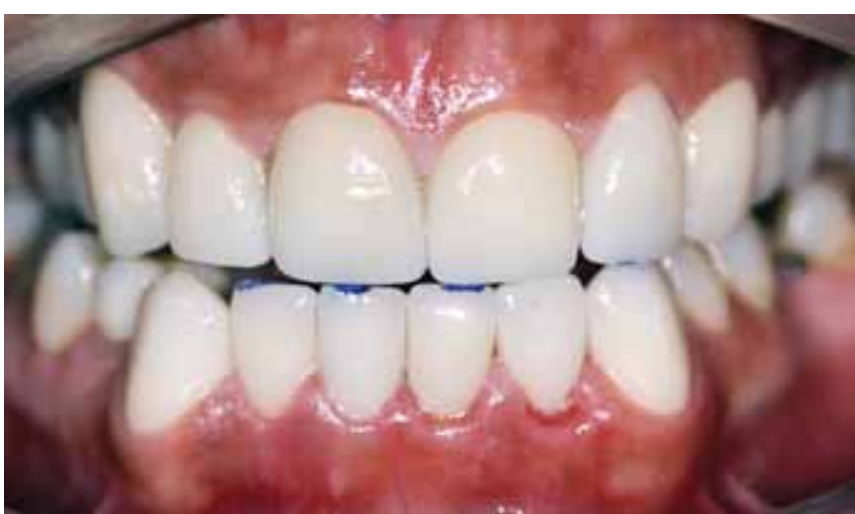

Fig. 6: Occlusion checked in centric and eccentric positions

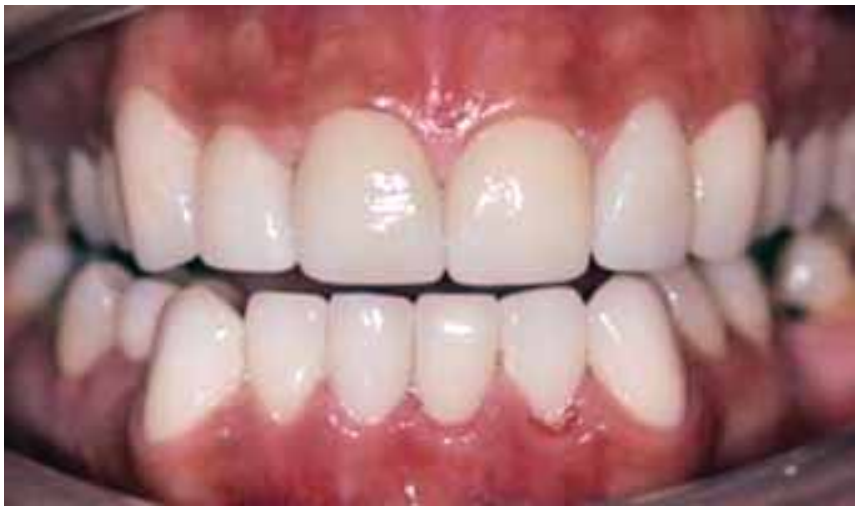

Fig. 7: Final view—-three days postcementation
The final result showed that the definitive restoration was esthetic, maintaining form, function and harmony while keeping in mind the patient goals (Fig. 7).

\section{CONCLUSION}

All ceramic restorations are extremely popular and have been used for many years with success. Dental lasers are a very useful tool in the hand of the skilled operator inasmuch it is minimally invasive, reduces patient discomfort, therefore increases patient acceptance. This article demonstrates the combined use of all ceramic restorations and dental lasers to successfully address the esthetic concerns of a patient.

\section{REFERENCES}

1. Lowe RA. Clinical use of the Er,Cr:YSGG laser for osseous crown lengthening: Redefining the standard of care.

2. Gurel G. The science and art of porcelain laminate veneers. Carol Stream IL: Quintessence Publishing Company 2003.

3. Stabholz A, Zeltser R, Sela M, et al. The use of lasers in dentistry: Principles of operation and clinical applications. Com-pend Contin Educ Dent 2003; 24:935-48.

4. Rosenberg ES, Cho SC, Garber DA. Crown lengthening revisited. Compend Contin Educ Dent 1990;20(6):527-42.

5. Lee EA. Laser-assisted gingival tissue procedures in esthetic dentistry. Pract Proced Aesthet Dent 2006;18(9):2-6. 